\title{
Unraveling the Origin of the Apparent Charge of Zwitterionic Lipid Layers
}

\author{
Lisa B. Dreier, ${ }^{\dagger \dagger}$ Amanuel Wolde-Kidan, ${ }^{\S}$ Douwe Jan Bonthuis, ${ }^{\|}$Roland R. Netz, ${ }^{\S}$ \\ Ellen H.G. Backus, ${ }^{\dagger, \perp}$ and Mischa Bonn* ${ }^{*} \dagger$ (1) \\ ${ }^{\dagger}$ Max Planck Institute for Polymer Research, Ackermannweg 10, 55128 Mainz, Germany \\ ${ }^{\ddagger}$ Graduate School Materials Science in Mainz, Staudingerweg 9, 55128 Mainz, Germany \\ ${ }^{\S}$ Fachbereich Physik, Freie Universität Berlin, Arnimallee 14, 14195 Berlin, Germany \\ "Institute of Theoretical and Computational Physics, Graz University of Technology, 8010 Graz, Austria \\ ${ }^{\perp}$ Department of Physical Chemistry, University of Vienna, Währinger Strasse 42, 1090 Vienna, Austria
}

Supporting Information

ABSTRACT: The structure of water molecules in contact with zwitterionic lipid molecules is of great biological relevance, because biological membranes are largely composed of such lipids. The interaction of the interfacial water molecules with the amphiphilic lipid molecules drives the formation of membranes and greatly influences various processes at the membrane surface, as the field that arises from the aligned interfacial water molecules masks the charges of the lipid headgroups from the approaching metabolites. To increase our understanding of the influence of water molecules on biological processes we study their structure at the interface using sumfrequency generation spectroscopy and molecular dynamics simulations. Interestingly, we find that water molecules at zwitterionic lipid molecules are mainly oriented by the field arising between the two oppositely charged molecular moieties within the lipid headgroups.

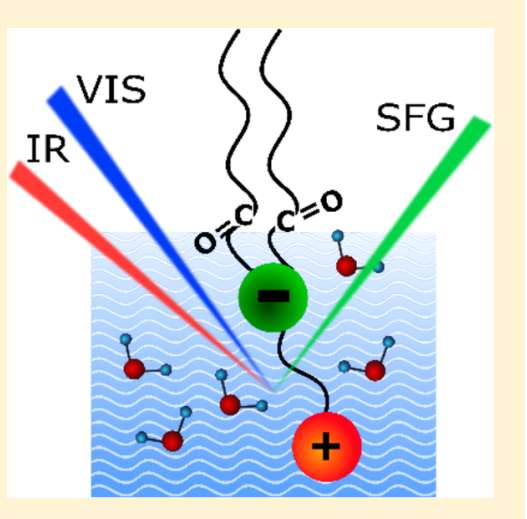

$\mathrm{T}$ he formation of membranes originates from the interaction of amphiphilic phospholipids with the adjacent water molecules. Hydrophobic forces drive the segregation of the hydrophobic tails and hydrophilic headgroups. ${ }^{1}$ Consequently, this interface between the lipid headgroups and the surrounding water molecules is of high biological relevance. Water molecules close to the lipid headgroups form strong hydrogen bonds to the phosphate and carbonyl groups of the lipid molecules. ${ }^{2,3}$

Furthermore, the water molecules are aligned because of the electric field arising from the charges on the lipid headgroups. ${ }^{4,5}$ Lipids bearing zwitterionic headgroups that have a net zero charge, such as phosphatidylcholine (PC) and phosphatidyl-ethanolamine (PE), are the main constituents of biological membranes. ${ }^{6}$ Interestingly, even though they have no net charge, the water molecules at the interface of such zwitterionic lipid layers have been concluded to be preferentially oriented, on average, with their hydrogen atoms pointing toward the lipid alkyl chain. ${ }^{5,7-9}$ Thus, the interfacial water molecules at such naturally occurring zwitterionic lipid surfaces orient as if the lipid were negatively charged. This water orientation, which is strongly enhanced as compared to the ordering at the water-air interface, has been shown experimentally with phase-resolved sum-frequency generation $(\mathrm{SFG})^{5}$ and theoretically using molecular dynamics (MD) simulations. ${ }^{10}$ Likewise, the zeta potential for liposomes of zwitterionic lipids has been reported to be negative at $\mathrm{pH}$
$7 .^{11}$ The water orientation and zeta potential thus both imply a preferential interfacial water orientation with the hydrogen atoms of the water molecules pointing toward the lipid monolayer. Throughout this Letter we will call this effect the "apparent negative charge of zwitterionic lipids", as the water orientation at these interfaces resembles that at negatively charged surfaces. This has important implications for biological reactions as it influences the apparent charge density of the surface, which in turn influences the interaction of membranes with water and proteins.

Various hypotheses have been put forward as to why water near naturally occurring zwitterionic headgroups behaves similarly to water close to negatively charged headgroups. One theory is that the water orientation is dominated by the negatively charged phosphate group that is more capable of orienting the water molecules because of its negative charge density being larger than the positive charge density of the choline group. ${ }^{8}$ However, there are also reports suggesting that the hydrogen bond network reaches up until the carbonyl groups and that those groups significantly contribute to the water-ordering driving force. ${ }^{7}$ In this scenario, it is simply the presence of carbonyl groups in the lipid headgroup that results

Received: September 4, 2019

Accepted: September 30, 2019

Published: September 30, 2019 
in the preferential orientation of interfacial water. Yet a third theory suggests that the dipole within the headgroup dominates the water response. In a lipid monolayer on water, the zwitterionic phosphatidylcholine headgroup is oriented in such a way that the phosphate group is closer to air, while the choline group is positioned a bit deeper in the water phase. ${ }^{9}$ This conformation results in the formation of an electric field between the negatively and positively charged molecular moieties. It has been suggested that this field significantly contributes to the interfacial water ordering. According to this theory, the water molecules situated between the lipid headgroups would thus experience the negative charge of the phosphate group from above and the positive charge of the choline group from below. ${ }^{5,9,12}$ This results in the water molecules being oriented with their hydrogen atoms pointing up with respect to the surface normal (toward the lipid monolayer).

Here, we unravel the origin of the apparent negative charge of zwitterionic lipids using the nonlinear optical method SFG spectroscopy. In SFG, a visible and an IR pulse are overlapped at the sample surface and a signal at the sum of those two frequencies is generated. If the IR pulse frequency is in resonance with a molecular vibration, the signal is enhanced. An SFG signal can be generated only at the surface of water where the symmetry is broken and is thus sensitive to the alignment of interfacial molecules. We can determine the origin of the apparent negative charge of naturally occurring zwitterionic lipid monolayers by investigating the water orientation at lipid monolayers, for which the chemical structure of the headgroup is altered such that the arrangement of the positive and negative charge are inverted. The zeta potential for liposomes of such zwitterionic lipids with altered headgroups has been reported to be negative at $\mathrm{pH} 7$ for both 1,2-dioleoyl-sn-glycero-3-phosphocholine (DOPC) and the zwitterionic lipid with the inverted headgroup 2-((2,3bis(oleoyloxy)propyl)-dimethyl-ammonio)ethyl ethyl phosphate (DOCPe). ${ }^{11}$ This suggests that the apparent charge and thus the interfacial water structure at those two lipids might be comparable. We thus investigate the water orientation for the two types of headgroups with altered phosphate and choline group sequence. We have measured SFG spectra of DOPC and DOCPe monolayers on water to determine the water orientation at those different zwitterionic lipid molecules.

Figure 1a shows the chemical structure of the two zwitterionic lipid molecules DOPC and DOCPe. The SFG spectra of the lipid-water interface for the two different lipids are shown in Figure $1 \mathrm{~b}$. The sharp signals between 2800 and $3000 \mathrm{~cm}^{-1}$ originate from $\mathrm{CH}$ vibrations of the lipid tails, and the broad signals between 3100 and $3500 \mathrm{~cm}^{-1}$ arise from oriented water molecules at the lipid monolayers. The spectral shape in the $\mathrm{OH}$ vibration region appears to be almost identical for the two lipid monolayers. The only differences occur in the $\mathrm{CH}$ signals of the lipid tails. This variation is due to the different surface pressures and to the consequentially different ordering of the monolayers on the water surface. The similarity in the water signals is remarkable, as an orientational reversal of water going from the $\mathrm{PC}$ to $\mathrm{CPe}$ zwitterionic headgroup has been found previously in $\mathrm{MD}$ simulations. ${ }^{12}$

Because the signal measured with conventional SFG spectroscopy is proportional to $\left|\chi^{(2)}\right|^{2}$, the information about the absolute orientation of the interfacial water molecules is lost. It is thus not possible to infer from these spectra whether

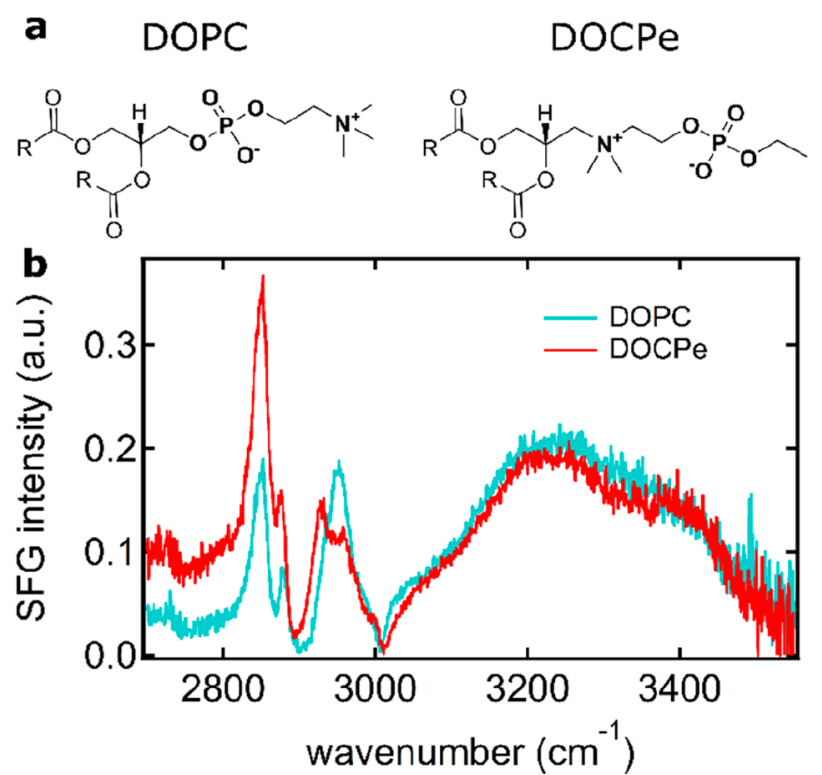

Figure 1. (a) Chemical structures of the zwitterionic lipid molecules DOPC (left) and DOCPe (right). (b) SFG spectra of the $\mathrm{H}_{2} \mathrm{O}$-lipid interface for the two different lipid molecules at a surface area per molecule of $80 \AA^{2}$.

the water molecules are oriented with their hydrogen atoms pointing up or down relative to the surface normal. We therefore also measured phase-resolved SFG spectra of the DOPC and DOCPe lipid monolayers on water. Figure 2 shows

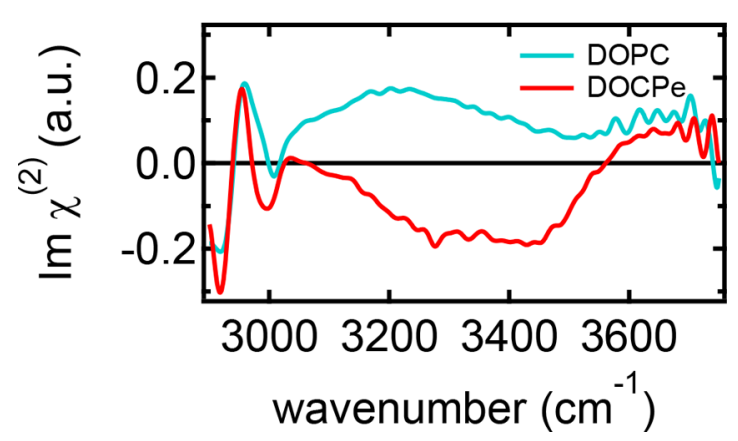

Figure 2. $\operatorname{Im} \chi^{(2)}$ spectra of the $\mathrm{H}_{2} \mathrm{O}$-lipid interface for the two different lipid molecules acquired at a surface area per molecule of 85 $\AA^{2}$.

the imaginary $\left(\operatorname{Im} \chi^{(2)}\right)$ SFG spectra of the lipid-water interface for the two different lipids. The spectra are acquired in ssp polarization combination. The sharp signals between 2900 and $3000 \mathrm{~cm}^{-1}$ originate from $\mathrm{CH}$ vibrations of the lipid tails. In accordance with previous literature, ${ }^{13}$ we assign the negative peak at $2920 \mathrm{~cm}^{-1}$ to a $\mathrm{CH}_{3}$ Fermi resonance and the positive peak at $2955 \mathrm{~cm}^{-1}$ to the $\mathrm{CH}_{3}$ asymmetric stretch mode. These signs of the $\mathrm{CH}_{3}$ resonances indicate that the methyl groups are oriented with their $\mathrm{H}$ atoms pointing up toward air. ${ }^{8,14}$ The negative signal at $3000 \mathrm{~cm}^{-1}$ is assigned to a vinyl $\mathrm{CH}$ stretch mode from the double bonds in the lipid chains. ${ }^{15}$ The broad signals between 3100 and $3700 \mathrm{~cm}^{-1}$ arise from oriented water molecules at the lipid monolayer surfaces. The sign of the main water signal is opposite for the two different zwitterionic lipids. A positive/negative water signal indicates that the water molecules are oriented with their dipoles pointing up/down. That is to say that the interfacial 

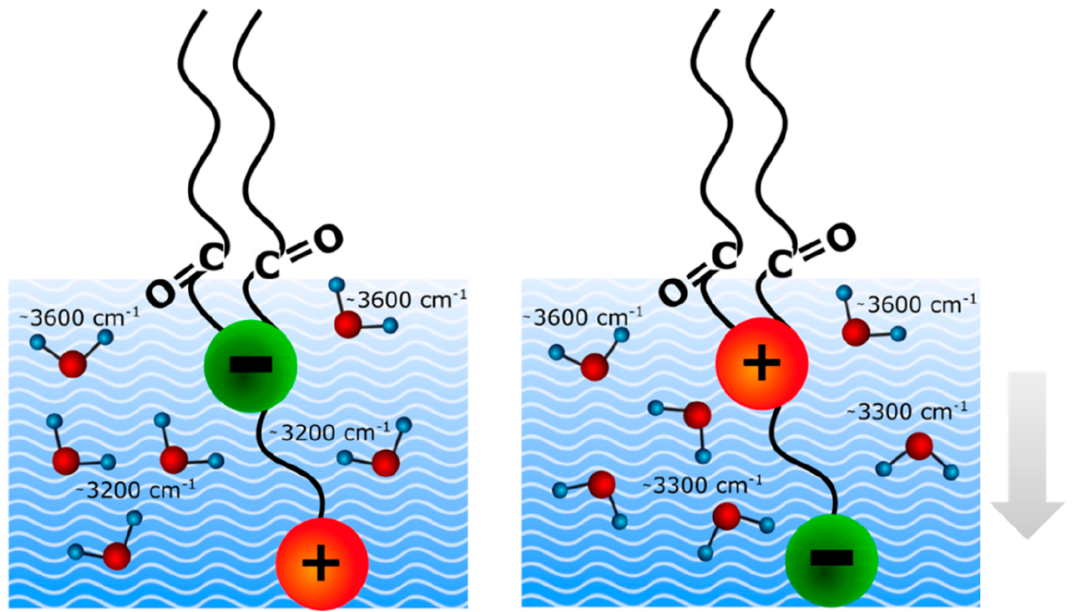

Figure 3. Schematic of a PC (left) and CPe (right) lipid and the water structure at the respective headgroups.
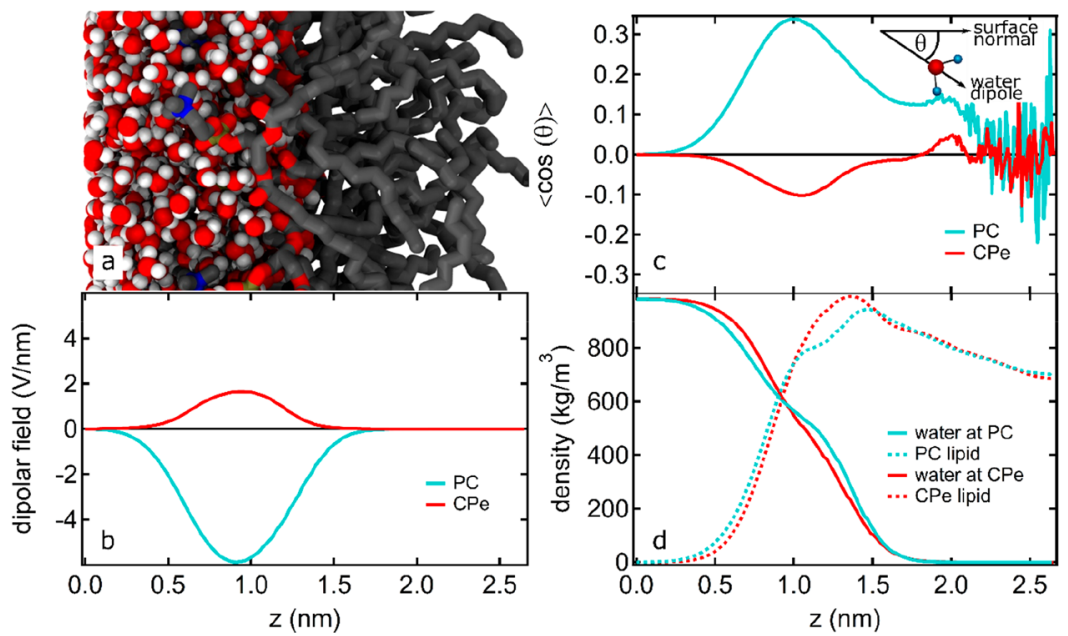

Figure 4. Results from molecular dynamics simulations of DOPC and DOCPe lipid bilayers in water. (a) Cutout of a snapshot of the simulated system for the DOPC bilayer. (b) Dipolar field profiles due to the oriented water molecules for DOPC (blue) and DOCPe (red). (c) Ordering profile of the water dipole moments along the surface normal for DOPC (blue) and DOCPe (red). $\cos (\theta)$ is defined as the angle between the water dipole moment and the surface normal (see inset in panel c). The water orientation at DOCPe is opposite from the DOPC case. Positive (negative) $\cos (\theta)$ indicates water with its $\mathrm{H}$ atoms oriented toward (away from) the lipid alkyl chain. (d) Density distributions of the lipids (dashed lines) and water (solid lines) molecules across the simulation box, shown for the DOPC (blue) and DOCPe (red) system.

water molecules are oriented with their hydrogen atoms pointing up/down relative to the surface normal (toward/away from the lipid monolayer). The positive SFG water signal at zwitterionic PC headgroups, i.e., as if the PC monolayer carried a net negative charge, has been reported previously. $5,7,8$

The main SFG water signal at the CPe headgroup is negative, indicating that the water molecules are mainly oriented with their hydrogen atoms pointing down toward the bulk water. As we observe a flip in the water orientation at the $\mathrm{CPe}$ monolayer as opposed to the PC monolayer, the preferential water orientation can not be induced by the stronger water ordering capability of the phosphate group that is present in both headgroups. Rather, it is the relative positions of the charged groups within the lipid headgroup that matter. Our results are therefore consistent with the theory that the electric field formed by the two charges in the zwitterionic headgroup has a strong impact on the interfacial water orientation. ${ }^{5,9,12}$ As the relative position of the phosphate and choline groups is interchanged for the two different lipid structures of the PC and CPe headgroups, the direction of the resulting field is likewise reversed. As a result, the interfacial water molecules are oriented in opposite directions for the two different headgroup structures. The position of the main water band at the DOPC monolayer is at a significantly lower frequency than at the DOCPe monolayer, indicating differences in the hydrogen bonding strength between the two interfaces. However, the results might also be induced by coupling effects. To determine the origin of the shift in the water band the phase-resolved experiments would have to be performed in isotopically diluted water, which is experimentally challenging because of the small signal strength.

The high-frequency $\left(\sim 3600 \mathrm{~cm}^{-1}\right)$ water signal is positive for both the PC and CPe headgroups. It thus originates from water molecules oriented with their hydrogen atoms pointing up in both cases. This high-frequency signal at zwitterionic lipid monolayers has been reported previously. ${ }^{7,8,16}$ It has been attributed to water molecules close to the lipid carbonyl groups $^{7}$ and in the hydrophobic region of the lipid molecules. ${ }^{8,16}$ As this high-frequency signal originates from water molecules that are not situated between the two charge 
centers, the structure of the charged groups in the headgroups does not influence the orientation of these water molecules. Thus, the signal is present in the spectra of the PC as well as the CPe headgroups. A schematic of the two lipid molecules and the ordered interfacial water molecules is shown in Figure 3.

To further investigate our experimental findings, we performed simulations of lipid bilayers in water using PC and $\mathrm{CPe}$ headgroups. The interactions at hydrated lipid bilayers are comparable to those at lipid monolayers. ${ }^{17}$ Figure 4a shows the cutout of a snapshot of the simulation for the $\mathrm{PC}-$ water case. The water orientation profiles along the surface normal at the DOPC and DOCPe interface are shown in Figure $4 c$. Figure $4 d$ shows the density distributions of the DOPC and DOCPe lipid molecules (dashed lines) as well as those of the water molecules (solid lines) as a function of the position in the simulation box. The dipolar electric fields arising from the oriented interfacial water molecules are shown in Figure $4 \mathrm{~b}$ for DOPC (blue) and DOCPe (red). Panels b and $c$ of Figure 4 show that the water molecules are oriented only in the headgroup region and not by a field that penetrates into the water as we have shown previously for charged lipid headgroups. ${ }^{18}$ The simulation results also show that the dipolar electric field arising from the interfacial water molecules is opposite for the two different lipid headgroups, which is in line with our experimental results. However, the intensity of the dipolar field arising from the interfacial water molecules (Figure 4b) is rather different for the two different lipids. This is in contrast to our experimental results, where we observe a comparable intensity of the SFG water signal for both systems (Figures $1 \mathrm{~b}$ and 2). This discrepancy might well originate from the force field used in the simulations.

Interestingly, there is a small fraction of water molecules at the DOCPe headgroup that are oriented in the opposite direction as the majority of water molecules (Figure 4c). These water molecules are situated closer to the lipid tails in the simulation box (roughly at $\pm 2 \mathrm{~nm}$ ). As water molecules closer to the hydrophobic tails are likely to be only loosely hydrogen bonded, these water molecules probably correspond to those water molecules that contribute to the positive high-frequency signal that is present in the spectra of both lipids shown in Figure 2. However, as the water density at this position in the simulation box is already quite low (Figure $4 \mathrm{~d}$ ), this positive signal is not present anymore in the calculated dipolar field shown in Figure $4 \mathrm{~b}$. This might again be due to the force field used in the simulations.

Previously reported theoretical results for the water orientation at zwitterionic lipids with inverted headgroups all show that the main water population is oriented oppositely at the $\mathrm{CPe}$ bilayer surface as compared to the PC bilayer surface, ${ }^{12,19,20}$ which is in agreement with our results. However, while the results all agree in terms of the average interfacial water orientation, there are quite some differences in the details. Magarkar et al. ${ }^{12}$ and Sani et al. ${ }^{19}$ both report a comparable intensity for the water orientation at the PC and $\mathrm{CPe}$ bilayer, while the results of Cardenas et al. ${ }^{20}$ show a significantly higher water ordering at the PC membrane than at the $\mathrm{CPe}$ membrane. Furthermore, the results differ quite drastically in the water orientation closer to the hydrophobic part of the membrane. Magarkar et al. ${ }^{12}$ and Cardenas et al. ${ }^{20}$ both observe a small population of water with reversed orientation at the $\mathrm{CPe}$ interface close to the membrane center. This is in agreement with our experimental (Figure 2) and theoretical (Figure 4c) results. Sani et al. ${ }^{19}$ on the other hand observe water with opposite orientation at this small distance to the membrane center. These differences in the simulation results may be due to the presence or absence of ions ${ }^{20}$ as well as the use of different force fields.

This inversion in the water orientation that we observe experimentally and in MD simulations for the zwitterionic lipid headgroups with different arrangements of the charged groups is not reflected in the previously reported zeta potential measurements mentioned above. ${ }^{11}$ This is not entirely surprising as the zeta potential reports the potential at the shear plane, ${ }^{21}$ which is significantly further away from the lipid headgroups than the water molecules that are situated between the lipid headgroups that contribute to our SFG signals and to the dipolar field in the MD simulations. Moreover, water dipoles do not contribute to the potential, so that charge from impurities adsorbed to the surface may be responsible for the negative zeta potential. ${ }^{22}$

In conclusion, we have shown that the relative position of the phosphate and choline moieties within the lipid headgroups in zwitterionic lipid monolayers determines the interfacial water orientation. Water molecules orient with their hydrogen atoms toward the PC alkyl chain while they orient with their hydrogen atoms pointing away from the $\mathrm{CPe}$ alkyl chain. These results suggest that water ordering induced by zwitterionic lipids is not dominated by the stronger water ordering capability of the phosphate group but rather the electric field that arises between the two oppositely charged groups within the headgroup. The water molecules residing close to the hydrophobic tails are not influenced by the inversion of the charge centers within the headgroups.

\section{ASSOCIATED CONTENT}

\section{S Supporting Information}

The Supporting Information is available free of charge on the ACS Publications website at DOI: 10.1021/acs.jpclett.9b02587.

Description of the experimental procedures and details of the simulation conditions (PDF)

\section{AUTHOR INFORMATION}

ORCID $\odot$

Ellen H.G. Backus: 0000-0002-6202-0280

Mischa Bonn: 0000-0001-6851-8453

\section{Notes}

The authors declare no competing financial interest.

\section{ACKNOWLEDGMENTS}

The authors thank Sudipta Das for an introduction to the phase-resolved setup and Kevin Machel for his help with the sample preparation. This work is partially funded by a DFG grant (BA 5008/3). L.B.D is a recipient of a position funded by the Deutsche Forschungsgemeinschaft through the Excellence Initiative by the Graduate School Materials Science in Mainz (GSC 266). We are grateful to the MaxWater initiative from the Max Planck Society for financial support.

\section{REFERENCES}

(1) Fraxelas, J. Water at Interfaces a Molecular Approach; CRC Press Taylor and Francis Group: Boca Raton, FL, 2014.

(2) Hübner, W.; Blume, A. Interactions at the Lipid-Water Interface. Chem. Phys. Lipids 1998, 96, 99-123. 
(3) Pohle, W.; Selle, C.; Fritzsche, H.; Binder, H. Fourier Transform Infrared Spectroscopy as a Probe for the Study of the Hydration of Lipid Self-Assemblies. I. Methodology and General Phenomena. Biospectroscopy 1998, 4, 267-280.

(4) Sung, W.; Seok, S.; Kim, D.; Tian, C. S.; Shen, Y. R. SumFrequency Spectroscopic Study of Langmuir Monolayers of Lipids Having Oppositely Charged Headgroups. Langmuir 2010, 26 (23), $18266-72$.

(5) Chen, X.; Hua, W.; Huang, Z.; Allen, H. C. Interfacial Water Structure Associated with Phospholipid Membranes Studied by Phase-Sensitive Vibrational Sum Frequency Generation Spectroscopy. J. Am. Chem. Soc. 2010, 132, 11336-11342.

(6) van Meer, G.; Voelker, D. R.; Feigenson, G. W. Membrane Lipids: Where They Are and How They Behave. Nat. Rev. Mol. Cell Biol. 2008, 9 (2), 112-24.

(7) Ohto, T.; Backus, E. H. G.; Hsieh, C. S.; Sulpizi, M.; Bonn, M.; Nagata, Y. Lipid Carbonyl Groups Terminate the Hydrogen Bond Network of Membrane-Bound Water. J. Phys. Chem. Lett. 2015, 6 (22), 4499-503.

(8) Mondal, J. A.; Nihonyanagi, S.; Yamaguchi, S.; Tahara, T. Three Distinct Water Structures at a Zwitterionic Lipid/Water Interface Revealed by Heterodyne-Detected Vibrational Sum Frequency Generation. J. Am. Chem. Soc. 2012, 134 (18), 7842-50.

(9) Ishiyama, T.; Terada, D.; Morita, A. Hydrogen-Bonding Structure at Zwitterionic Lipid/Water Interface. J. Phys. Chem. Lett. 2016, 7 (2), 216-20.

(10) Marrink, S.-J.; Berkowitz, M.; Berendsen, H. Molecular Dynamics Simulation of a Membrane/Water Interface: The Ordering of Water and Its Relation to the Hydration Force. Langmuir 1993, 9, $3122-3131$.

(11) Perttu, E. K.; Kohli, A. G.; Szoka, F. C., Jr. InversePhosphocholine Lipids: A Remix of a Common Phospholipid. J. Am. Chem. Soc. 2012, 134 (10), 4485-8.

(12) Magarkar, A.; Róg, T.; Bunker, A. Molecular Dynamics Simulation of Inverse-Phosphocholine Lipids. J. Phys. Chem. C 2014, 118 (33), 19444-19449.

(13) Okuno, M.; Mezger, M.; Stangenberg, R.; Baumgarten, M.; Mullen, K.; Bonn, M.; Backus, E. H. G. Interaction of a Patterned Amphiphilic Polyphenylene Dendrimer with a Lipid Monolayer: Electrostatic Interactions Dominate. Langmuir 2015, 31 (6), 1980-7. (14) Ji, N.; Ostroverkhov, V.; Chen, C.-Y.; Shen, Y.-R. PhaseSensitive Sum-Frequency Vibrational Spectroscopy and Its Application to Studies of Interfacial Alkyl Chains. J. Am. Chem. Soc. 2007, 129, 10056-10057.

(15) Qiao, L.; Ge, A.; Osawa, M.; Ye, S. Structure and Stability Studies of Mixed Monolayers of Saturated and Unsaturated Phospholipids under Low-Level Ozone. Phys. Chem. Chem. Phys. 2013, 15 (41), 17775-85.

(16) Ma, G.; Chen, X.; Allen, H. C. Dangling OD Confined in a Langmuir Monolayer. J. Am. Chem. Soc. 2007, 129, 14053-14057.

(17) Stefaniu, C.; Brezesinski, G.; Mohwald, H. Langmuir Monolayers as Models to Study Processes at Membrane Surfaces. Adv. Colloid Interface Sci. 2014, 208, 197-213.

(18) Dreier, L. B.; Nagata, Y.; Lutz, H.; Gonella, G.; Hunger, J.; Backus, E. H. G.; Bonn, M. Saturation of Charge-Induced Water Alignment at Model Membrane Surfaces. Sci. Adv. 2018, 4, No. eaap7415.

(19) Rezaei Sani, S. M.; Akhavan, M.; Jalili, S. Salt-Induced Effects on Natural and Inverse DPPC Lipid Membranes: Molecular Dynamics Simulation. Biophys. Chem. 2018, 239, 7-15.

(20) Cardenas, A. E.; Anderson, C. M.; Elber, R.; Webb, L. J. Partition of Positively and Negatively Charged Tryptophan Ions in Membranes with Inverted Phospholipid Heads: Simulations and Experiments. J. Phys. Chem. B 2019, 123 (15), 3272-3281.

(21) Smith, M. C.; Crist, R. M.; Clogston, J. D.; McNeil, S. E. Zeta Potential: A Case Study of Cationic, Anionic, and Neutral Liposomes. Anal. Bioanal. Chem. 2017, 409 (24), 5779-5787.
(22) Bonthuis, D. J.; Horinek, D.; Bocquet, L.; Netz, R. R. Electrokinetics at Aqueous Interfaces without Mobile Charges. Langmuir 2010, 26 (15), 12614-12625. 